\title{
Sex hormones play roles in determining musk composition during the early stages of musk secretion by musk deer (Moschus berezovskii)
}

\author{
Mengyuan $\mathrm{Fan}^{1)}{ }^{*}$, Meishan Zhang ${ }^{1)}$, Minghui Shi ${ }^{1)}$, Tianxiang Zhang ${ }^{1)}$, Lei Qi ${ }^{1)}$, Juan $\mathrm{Yu}^{2)}$, Xuxin $\mathrm{Li}^{2)}$, \\ Shaobi Lin ${ }^{2}$, Zhixin Huang ${ }^{2}$, Shuang Yang ${ }^{1)}$, Juntong Zhou ${ }^{1)}$, Yimeng Li ${ }^{1)}$, Xiaoning Sun ${ }^{1)}$, Muha Cha ${ }^{1)}$, \\ Shanghua $\mathrm{Xu}^{1)}$, Yang Liu ${ }^{1)}$, Xiaobing $\mathrm{Guo}^{1)}$, Defu $\mathrm{Hu}^{1)}$ and Shuqiang Liu ${ }^{1), 2)}$ \\ 1) College of Nature Conservation, Beijing Forestry University, Beijing 100083, People's Republic of China \\ 2) Zhangzhou Pien Tze Huang Pharmaceutical Co., Ltd., Fujian 363700, People's Republic of China
}

\begin{abstract}
Musk is a secreted external hormone or information compound that is stored in musk scent glands of the males of species within the family Moschidae, such as Moschus berezovskii. The secretion of musk changes periodically during the courtship and reproduction periods, with the early stage of secretion occurring from May to July, and the maturation stage occurring from August to April of the following year. In this study, we analyzed the dynamic changes in musk components from June to April of the following year. The result showed that musk morphological character, water content, total ion chromatographic pattern, and composition undergo seasonal change. Luminescence immunoassay and radioimmunoassay analyses were performed to determine corresponding fecal hormone levels. The results showed that testosterone, estrogen, and cortisol levels in feces change on a seasonal basis, and are significantly higher in June than in other months $(p<0.01)$. Correlation analysis showed that the contents of four examined musk components (muscone, cyclopentadecanone, cholesterol, and cholestenol) from June to August were significantly highly negatively correlated with fecal testosterone and estradiol levels $(p<0.01)$. In contrast, the correlation coefficients were low or not significant from August to April of the following year. These results indicate that testosterone and estradiol may play a major role in determining musk composition during the early stage of musk secretion but not during the course of musk maturation, which suggests that musk secretion may be promoted by increases in sex hormones in June.
\end{abstract}

Key words: Musk secretion, Musk component, Sex hormone, Musk deer

MUSK DEER are small Cervidae animals, species of which include the Chinese forest musk deer (Moschus

Submitted May 29, 2018; Accepted Jul. 21, 2018 as EJ18-0211

Released online in J-STAGE as advance publication Aug. 31, 2018

Correspondence to: Shuqiang Liu, 1 College of Nature Conservation, Beijing Forestry University, No.35, Qinghua East Road, Haidian District, Beijing 100083 People's Republic of China; 2 Zhangzhou Pien Tze Huang Pharmaceutical Co., Ltd., Fujian 363700, People's Republic of China.

E-mail: liushuqiang@bjfu.edu.cn

Correspondence to: Defu Hu, No. 35, Qinghua East Road, Haidian District, Beijing 100083, People's Republic of China.

E-mail: hudf@bjfu.edu.cn.

*These authors contributed equally to this work

Abbreviations: GC-MS: gas chromatography-mass spectrometry; RIA: radioimmunoassay; RLU: relative light units; NSB: nonspecific binding test; IQR: interquartile range berezovskii), the Alpine musk deer (M. sifanicus), and the Siberian musk deer (M. moschiferus). Due to habitat loss and indiscriminate poaching, wild musk deer populations are currently endangered (CITES, Annex II), and the species has a National Class I protected status [1]. Since the 1950s, musk deer have been artificially bred in China in an effort to restore the populations of this mammal.

Musk, a pheromone that is mainly used for communication, is secreted from the scent glands of mature males of different species of musk deer. In the Chinese forest musk deer, the oblate scent glands protrude from between the abdomen and the scrotum [2,3].

During the early phases of the musk secretion season, the scent glands gradually enlarge, their openings turn red, and musk fluid volume gradually increases. Behav- 
iorally, the deer become excitable and aggressive. During the peak of musk production period, males stop eating, decrease water intake, and move relatively little. At this time, the scent glands reach maximum volume, with thickened walls, enlarged subcutaneous blood vessels, and substantial fluid accumulation. This musk fluid is expelled through frequent contractions of the scent glands. Subsequently, males resume feeding, their scent glands shrink significantly, and the production of musk fluid is considerably reduced, during which time it gradually becomes brown, reddish-brown, or coffee colored as the water content decreases [2,4]. The musk secretion season of musk deer extends from May to July, whereas during the remainder of the year, no musk is secreted. In the breeding season, unmated male musk deer can use musk as a pheromone to attract females [5].

Studies on the quantitation of musk components have been limited by the complexity of these substances, and those that have been undertaken have not been performed systematically [6]. To date, there has been no systematic analysis that has accurately measured musk components across the different periods of musk maturation. Classic studies on musk have indicated that the major components are muscone, steroids, amino acids, and polypeptides [7]. Subsequent research has confirmed that androstane is one of the important components of musk [8]. Studies have also demonstrated that fatty acids and esters are other important constituents [9]. However, these studies have focused only on determining the composition of mature musk and have not attempted to analyze immature post-secretion musk fluid or its components. Nevertheless, the appearance and composition of musk undergo many changes from the onset of secretion to final maturation, and the current lack of data on the changes in musk components that occur during the maturation process has hindered an accurate determination of the dynamic changes in musk during maturation.

Analysis of the chemical composition of male musk deer serum during different periods has shown that the content of 17-ketosteroid, a metabolite of testosterone, increases significantly during the incipient stages of musk secretion. Removing the testis and epididymis of a normal male musk deer suppresses musk secretion, whereas the secretion ability can be restored by injection of exogenous testosterone [2]. These findings indicate that the secretion of musk by musk deer is closely related to endogenous testosterone levels. Notably, injections of exogenous testosterone can promote musk secretion during the non-secretion season $[10,11]$, and can even restore musk secretion in old musk deer that have otherwise lost this ability [12]. Injecting exogenous luteinizing hormone, luteinizing hormone-releasing hormone, follicle-stimulating hormone, and human chorionic gonadotropin can also promote musk secretion during the non-secretion season, although the effect is not as pronounced as that obtained with exogenous testosterone $[13,14]$. Significantly, however, the quantity of musk secretion stimulated in response to injecting with exogenous testosterone or gonadotropic hormones is less than $50 \%$ that of the naturally secreted musk [15], which indicates that secretion is not only affected by testosterone or other gonadotropic hormones but also involves complex physiological reactions regulated by a variety of hormones. Moreover, given that musk secretion undergoes periodic change concomitant with courtship and breeding, it is conceivable that changes in musk composition are related to seasonal variations in hormone levels. To date, however, there have been no analyses of the correlation between hormone levels and changes in the composition of musk.

Studies of musk deer hormone are, nevertheless, hampered by difficulties in collecting multiple blood samples, as these animals are highly alert, easily frightened, and avoid humans [16, 17]. In recent years, non-invasive sampling methods, such as the collection of feces, urine, saliva, and other secretions, have broadened the research routes available for the study of wildlife physiology [18]. For example, hormone quantitation using fresh fecal samples is considered to be a particularly useful technique [19]. Such non-invasive techniques have also proven to be helpful in study of the Chinese forest musk deer [20].

In this study, we analyzed the dynamic changes that occur in musk appearance and composition during the course of musk maturation. In addition, we used radioimmunoassays and luminescence immunoassays to determine hormone levels in feces. Studies have identified 2heptanone and squalene as two of the main components of pheromones in male rats [21], and that testosterone plays a role in pheromone regulation [22]. The odorant components regulated by testosterone and estradiol are often active constituents [23]; for example, muscone and cyclopentadecanone which are ketones, and cholesterol and cholestenol, which are derivatives of squalene. These four components may play roles during male musk deer breeding similar to those played by 2-heptanone and squalene in male rats. Accordingly, in this study, we analyzed levels of the four musk constituents muscone, 
cyclopentadecanone, cholesterol, and cholestenol to examine their correlation with hormone levels and the roles they may play in pheromones.

\section{Materials and Methods}

\section{Sample collection}

Ten healthy captive-bred adult male Chinese forest musk deer (6 years old) from the musk deer breeding base in Fengxian, Shanxi Province, were selected for collecting musk and feces samples at 2-month intervals during the period 2016-2017 (June 2016, August 2016, October 2016, December 2016, February 2017, and April 2017). Each deer was maintained in a separate captive facility.

Prior to collecting musk, the deer were placed in cages, and the area surrounding the secretion gland stoma and experimental instruments were sterilized with alcohol. The base of the gland was clamped and a curette was used to rapidly collect musk, after which the deer were released. Fresh feces was also collected from each deer immediately after it had been excreted.

After the removal of hair and other debris from the extracted musk and fecal samples, they were placed in sterile centrifuge tubes, labeled with details of the sampling time and subject's ear-tag number, and then stored frozen at $-20^{\circ} \mathrm{C}$.

\section{Quantitation of the water content in musk}

Empty centrifuge tubes were labeled, weighed, and recorded as $\mathrm{M}_{1}$. The tubes were then reweighed and recorded as $\mathrm{M}_{2}$ after the addition of musk $(<0.1 \mathrm{mg})$ and $100 \mu \mathrm{L}$ distilled water (the control contained water only). The tubes (with loosened caps) were then placed in a desiccator containing a mixture of sulfates of magnesium, calcium, and sodium. When the weight of the control tube became constant, sample tubes were reweighed and recorded as $\mathrm{M}_{3}$. The musk water content was accordingly calculated as follows: $P_{\text {water }}=\left(M_{2}-M_{3}\right) /\left(M_{2}-M_{1}\right)$.

\section{Quantitation of musk component levels}

The musk was dried in a dryer containing calcium sulfate. Thereafter, a 50-mg sample of dried musk was dissolved in $1 \mathrm{~mL}$ dichloromethane. After the mixture had been decanted into a disposable syringe, it was filtered through a micropore filter (Nylon, $25 \mathrm{~mm}, 0.22 \mu \mathrm{m}$; Jinteng, China) and used for gas chromatography-mass spectrometry (GC-MS) analysis. Two milliliters of the filtrate was placed in a sample bottle that was inserted into a GC-MS system (Model QP2010; Shimadzu Corp., Kyoto, Japan) for quantitation. The operating conditions were as follows: pre-column pressure: $60 \mathrm{kPa}$, splitless injection; inlet temperature: $250^{\circ} \mathrm{C}$; interface temperature: $280^{\circ} \mathrm{C}$; temperature programming: $80^{\circ} \mathrm{C}(1 \mathrm{~min})$, $+5^{\circ} \mathrm{C} \min ^{-1}$ to $245^{\circ} \mathrm{C}, 245^{\circ} \mathrm{C}(1 \mathrm{~min}),+10^{\circ} \mathrm{C} \min ^{-1}$ to $280^{\circ} \mathrm{C}$, and then maintained for $5 \mathrm{~min}$; carrier gas: He; sample volume: $1 \mu \mathrm{L}$; ion source temperature: $250^{\circ} \mathrm{C}$;

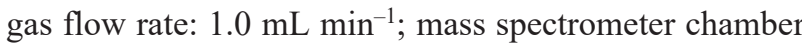
temperature: $230^{\circ} \mathrm{C}$; quadrupole analyzer temperature: $150^{\circ} \mathrm{C}$; electron impact energy: $70 \mathrm{eV}$; mass scanning range: 40.0-400.0 amu [6].

Post-analysis, the structures of fragmentation ions were compared with those in the mass spectral library of the National Institute of Standards and Technology. Chromatography retention times and other relevant data were also logged. Collectively, these data were used to verify musk components. Changes in the various components in the total ion chromatograms were compared with data from previous studies [6-9, 24]. For the purposes of analysis, we selected the following four musk components: muscone, cyclopentadecanone, cholesterol, and cholestanol. In order to determine variations in the selected components, area $\%$ values were obtained by dividing the area of each peak by the total area of the peaks of components contained in the same amount of musk.

\section{Quantitation of hormone levels in Chinese forest musk deer feces}

Fecal samples were milled, and $0.50 \mathrm{~g}$ subsamples were placed in $10-\mathrm{mL}$ centrifuge tubes, followed by the addition of $5 \mathrm{~mL} 90 \%(\mathrm{v} / \mathrm{v})$ ethanol. After agitation and extraction for $20 \mathrm{~min}$, the tubes were centrifuged for 20 $\min$ at $2,500 \mathrm{rpm}$ and the supernatants were collected. After the lower layers had precipitated, a further $5 \mathrm{~mL}$ $90 \%(\mathrm{v} / \mathrm{v})$ ethanol was added to each tube, followed by agitation and extraction for $20 \mathrm{~min}$, and then recentrifugation for $20 \mathrm{~min}$ at 2,500 rpm. Supernatants were decanted, combined with the previously prepared supernatants, and evaporated to dryness in a $60^{\circ} \mathrm{C}$ water bath. Thereafter, $1 \mathrm{~mL}$ of phosphate buffer solution was added to each tube, followed by agitation to recover the hormones. Tubes were stored at $-20^{\circ} \mathrm{C}$.

Fecal cortisol levels were quantified via RIA using a GC-2016 counter (Anhui Zonkia Scientific Instruments Co. Ltd., Anhui, China). The parameters recommended by the manufacturer of the cortisol reagent (Beijing North Institute of Biotechnology, Beijing, China) are as 
follows: sensitivity, $\leq 2.0 \mathrm{ng} \mathrm{mL}{ }^{-1}$; intra-batch coefficient of variation, $<10 \%$; inter-batch coefficient of variation, $<15 \%$.

The specific detection processes were as follows. Reagents and samples were maintained at room temperature for 15 30 min prior to use. These, together with 100 $\mu \mathrm{L}{ }^{125-}$ - -cortisol, were then added to the following polystyrene test tubes: total tube, nonspecific binding test (NSB) tube, blank tube, and standard tubes, In addition, $50 \mu \mathrm{L}$ standard $0\left(\mathrm{~S}_{0}\right)$ and $100 \mu \mathrm{L}$ distilled water were added to the NSB tube; $50 \mu \mathrm{L} \mathrm{S}_{0}$ and $100 \mu \mathrm{L}$ Cor. antibody were added to the blank tube; corresponding Cor. standards $\left(\mathrm{S}_{1}-\mathrm{S}_{5}\right)$ and $100 \mu \mathrm{L}$ Cor. antibody were added to the standard tubes; and $50 \mu \mathrm{L}$ samples and $100 \mu \mathrm{L}$ Cor. antibody were added to sample tubes. The tubes were shaken well, and then incubated at $37^{\circ} \mathrm{C}$ for $45 \mathrm{~min}$. With the exception of the total tube, $500 \mu \mathrm{L}$ immunosorbent was added to each tube. The tubes were shaken well, left to stand for $15 \mathrm{~min}$, and then centrifuged for 15 $\min$ at $3,500 \mathrm{rpm}$. The resulting supernatants were discarded and counts per minute in the precipitates were recorded.

Fecal testosterone and estradiol were quantified with luminescence immunoassays using a TZD-CL-200S reader (TZD Biotechnology, Fujian, China). The parameters recommended by the manufacturer of the testosterone and estradiol reagent kits (Beijing North Institute of Biotechnology, Beijing, China) are as follows: recovery rate, $90 \%-110 \%$; intra-analysis precision, $<15 \%$; intrabatch precision, $<20.0 \%$.

The specific detection processes were as follows. Reagents and samples were maintained at room temperature for 15 30 min before use. Standard or sample (25 $\mu \mathrm{L}$ ) was added to each well of 96-well microtiter plates, followed by the addition of $50 \mu \mathrm{L}$ enzyme conjugate. The plates were shaken well, and then incubated at $37^{\circ} \mathrm{C}$ for $1 \mathrm{~h}$. The liquid content in wells was then discarded and the wells washed. Thereafter, $100 \mu \mathrm{L}$ of mixed substrates was added to each well, and then after 5 to $10 \mathrm{~min}$ reacting in a light-tight container, relative light units (RLU) were measured. The estradiol results were analyzed by four-parameter fitting. A standard curve was prepared, with standard concentration along the X-axis, and RLU along the Y-axis. Using the standard curve, sample concentrations were determined from the measured RLU values. The testosterone results were analyzed by $\log$-logit linear fitting. A standard curve was prepared, with the logarithm of standard concentration along the $\mathrm{X}$-axis and logit values along the Y-axis. Sample con- centrations were determined from the measured RLU values, using the following equation:

$\operatorname{logit}=\ln \frac{B / B 0}{1-B / B 0}$,

where $\mathrm{B} 0$ is the RLU of $\mathrm{S}_{0}$, and $\mathrm{B}$ is the RLU of standards or samples.

\section{Data statistics and analysis}

Microsoft Excel 2013 software was used to organize the data. SPSS 25 was used to generate box plots of the monthly variations in musk water content, musk components, and fecal steroid hormone levels. Significant differences in hormone levels between two successive months were determined with generalized linear mixed models using SPSS 25. $P$ values of less than $5 \%$ were considered statistically significant. SPSS 25 was also used to perform Spearman correlation analyses of the various musk components and the corresponding fecal testosterone, estradiol, and cortisol levels for each sample and period. All tests were two-tailed and the significance level was set to $\alpha=0.05$.

\section{Results}

\section{Changes in musk morphology}

In June, the musk was a pale milky-white oily liquid containing small amounts of granular brown musk. By August, it had become a reddish-brown oily liquid or semi-solid. From October to April, the musk matured, becoming a brown solid (Fig. 1).

\section{Quantitation of water content in musk}

Musk water content was highest in June, decreased thereafter (Fig. 2), and remained at a relatively constant level from the beginning of December to the following April.

\section{GC-MS total ion-current chromatography of musk}

The results of the GC-MS total ion-current chromatography (Fig. 3) of musk components for each month shows that the number and area of peaks in June were small, but thereafter increased until December. From December to April, some peaks began to decline, and the peak area decreased. These results indicate that the variety and content of musk components increased from June to December and decreased from December to April of the following year, and also suggest that the variety and content of musk components changed during different 


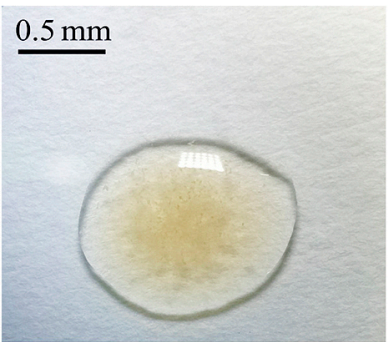

A

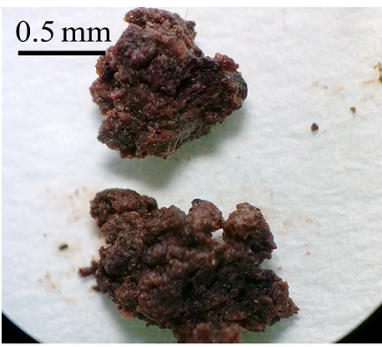

$\mathrm{D}$

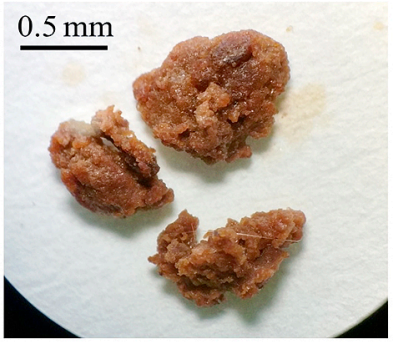

B

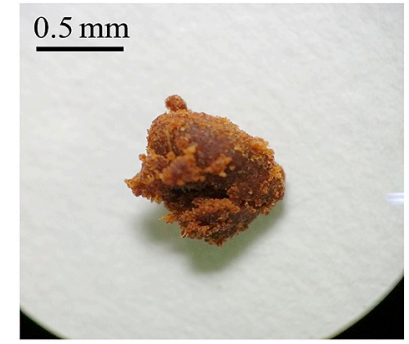

E

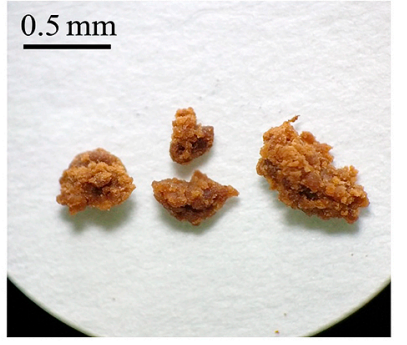

C

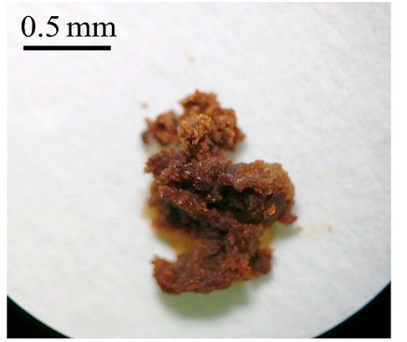

$\mathrm{F}$

Fig. 1 The external appearance of musk collected in different months during the period 2016-2017 (A: June 2016, B: August 2016, C: October 2016, D: December 2016, E: February 2017, F: April 2017).

periods of musk secretion.

\section{Quantitation of the levels of musk component}

On the basis of the GC-MS results, more than 100 components were identified in musk samples. Of the four musk components selected for analysis, muscone gradually increased from June to December, and then decreased thereafter, whereas cyclopentadecanone was not detected in June, but had increased by August, stabilizing in October and December, before gradually decreasing. Cholesterol was similarly undetectable in June, then gradually increased from August to December, decreased in February, and further decreased to zero in April. Levels of cholestanol showed a very similar pattern, being undetected in June, slowly increasing between August and December, and eventually declining to zero in February and April (Fig. 4).

\section{Quantitation of hormone levels in Chinese forest musk deer feces}

Analysis of the monthly hormonal variations indicated that testosterone levels changed significantly from June to August $(p<0.001)$, whereas they were low and stable during the remainder of the year (Fig. 5A). The same pattern was observed for estradiol (Fig. 5B). Cortisol levels were relatively high in June and gradually increased

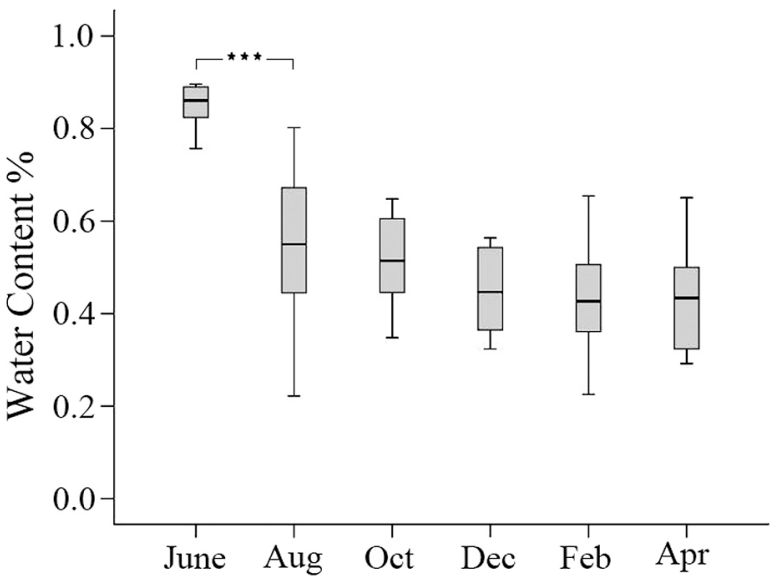

Fig. 2 Box plots of musk water content in different months. Xaxis: months (June 2016, August 2016, October 2016, December 2016, February 2017, April 2017) $\left(^{*}: p<0.05\right.$; $* *: p<0.01 ; * * *: p<0.001)$.

from August to December, before declining during February through April.

\section{Correlation between musk component levels and hormone levels}

From June to August, the four major components in musk were significantly highly negatively correlated $(p<$ 

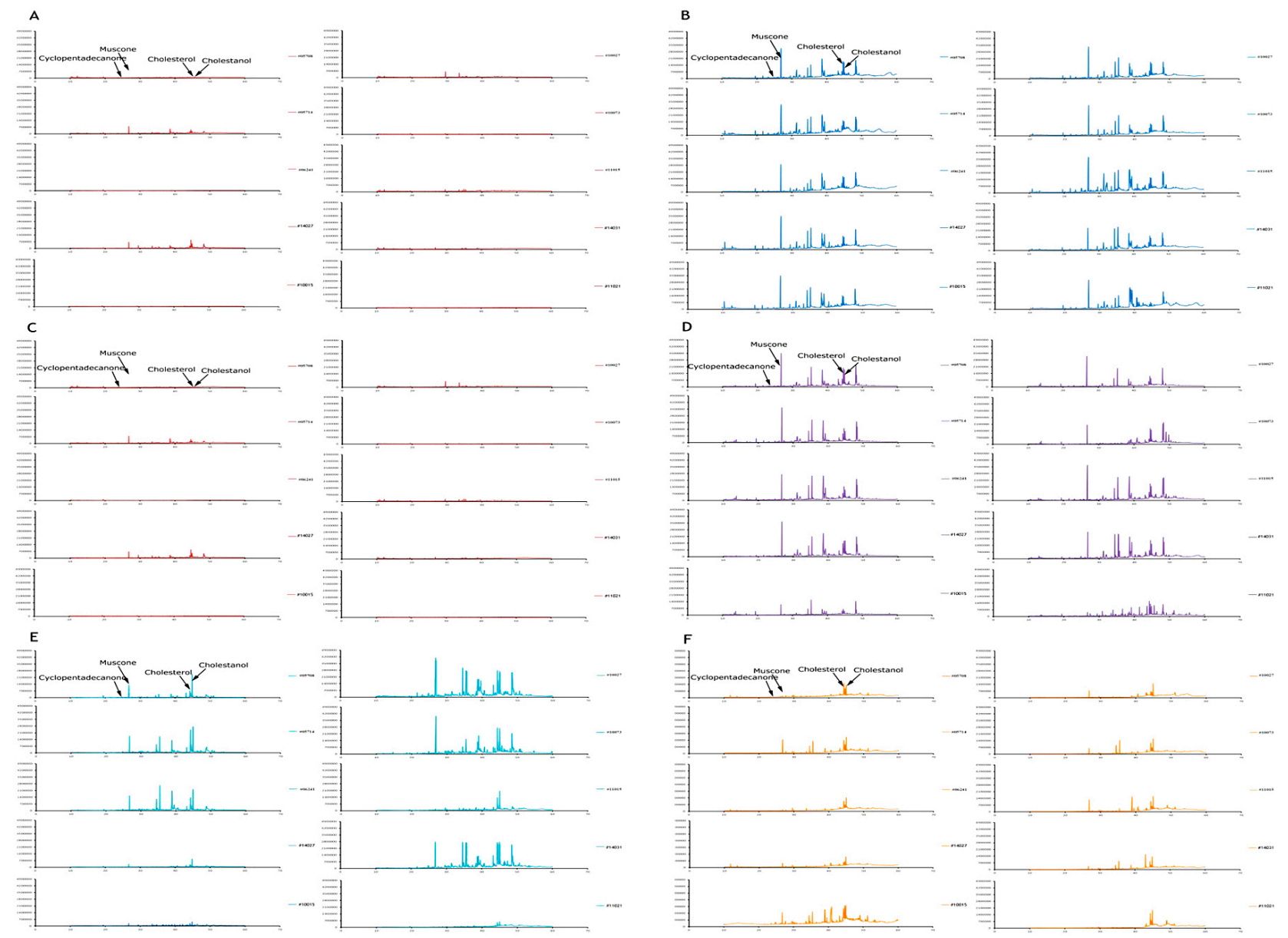

Fig. 3 Total ion chromatograms of GC-MS results from different months. X-axis: retention time; Y-axis: peak area. The right panel shows the ear-tag numbers of Chinese forest musk deer. Ten individuals per month. (A: June, B: August, C: October, D: December, E: February, F: April).

0.01 ) with fecal testosterone and estradiol levels (Tables 1A, 2A). From August to April of the following year, only cholesterol and cholestanol showed significant $(p<$ $0.05)$ positive correlations with fecal testosterone level (Tables 1B, 2B), and cholesterol and estradiol showed significant $(p<0.05)$ positive correlations. However, their correlation coefficients were low. From June to August and August to April of the following year, none of the four components were significantly correlated ( $p \geq$ 0.05) with fecal cortisol levels (Table 3).

\section{Discussion}

To the best of our knowledge, the present study is the first examine the changes in morphological character and water content of musk over the period from June of one year to April of the next. We observed that the form of musk changes from a pale milky-white oily liquid to a brown solid during different stages of the secretion period (Fig. 1). The water content was higher in June than in other months, and gradually decreased as the color of musk deepened to brown, with the content finally stabilizing by December (Fig. 2). These results indicated that moisture decreased during musk maturation, and musk turns from liquid to solid.

Chromatographic analysis of musk at different stages of maturation revealed that the contents of musk components undergo periodic change. In June, musk was characterized by few components and low contents, but these gradually increased until December, and declined slightly from December to April of the following year (Fig. 3). These observations reveal that the species and contents of musk compounds gradually increased over time. We detected a distinct component fingerprint from 


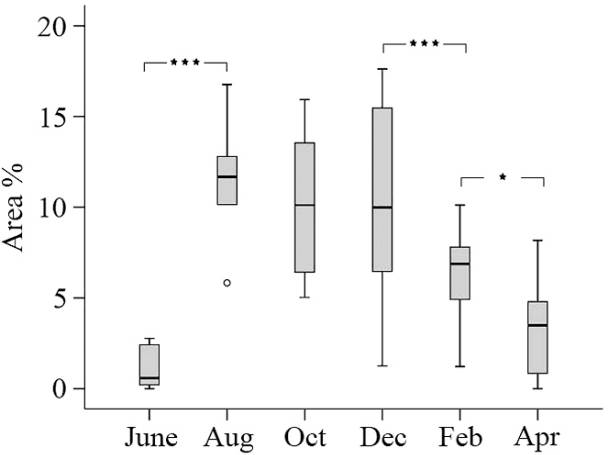

A. Muscone

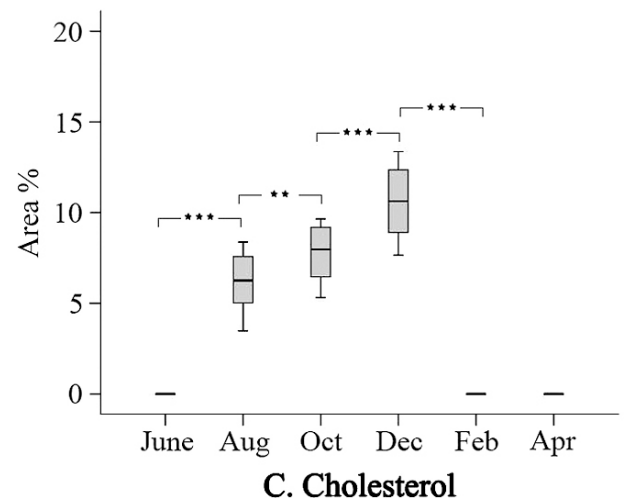

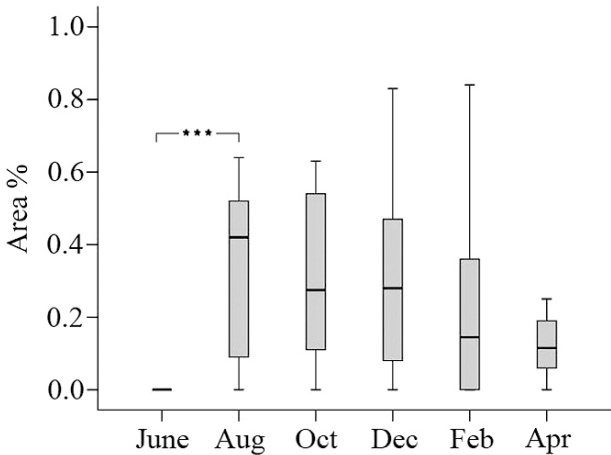

B. Cyclopentadecanone

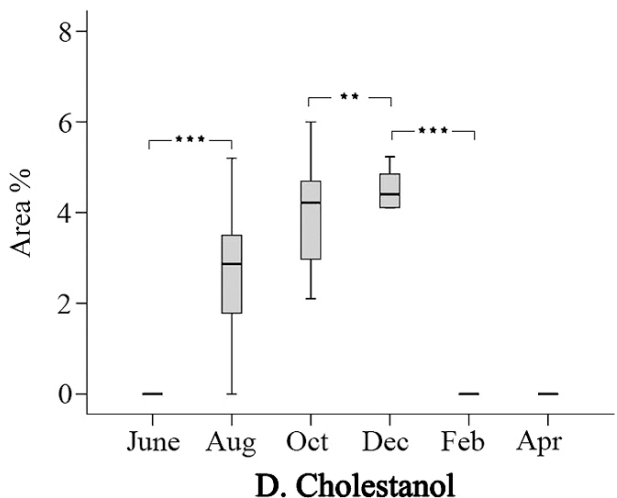

Fig. 4 Box plots of major musk component levels in different months. X-axis: months (June 2016, August 2016, October 2016, December 2016, February 2017, April 2017); Y-axis: corresponding component levels (\%) (A: Muscone, B: Cyclopentadecanone, $\mathrm{C}$ : Cholesterol, D: Cholestanol). ${ }^{\circ}$ indicates greater than 1.5 times and less than 3 times the interquartile range (IQR). $(*: p<0.05$; **: $p<0.01 ; * * *: p<0.001)$.

August to February, with that in December being the most obvious.

We selected four active pheromone constituents for analysis of correlations between changes in musk contents and hormone levels. Changes in the contents of these four components indicated that muscone, the primary component of musk, was highest in December, as were cyclopentadecanone, cholesterol, and cholestanol. In February, the contents of muscone and cyclopentadecanone decreased, and those of cholesterol and cholestanol declined to zero, which may be indicative of the initiation of a new annual cycle of musk secretion. On the basis of these results and the detection of other components by GC-MS, we hypothesized that cholesterol and cholestanol may have been converted to other substances at this time of year and conjecture that certain chemical reactions or mutual conversion may have occurred between these compounds (Fig. 4), possibly as a consequence of microbial fermentation.

To gain a better understanding of the endocrine mech- anisms underlying musk secretion, we used a noninvasive approach for the dynamic quantitation of fecal hormones in Chinese forest musk deer. Significant changes were observed in testosterone and estradiol levels, whereas in contrast, cortisol showed very little fluctuation (Tables 1-3). In June, which is a nonbreeding period, we observed significant differences in testosterone and estradiol levels in feces (Fig. 5), Similarly, previous studies have also shown that levels of fecal estradiol [25] and serum testosterone and estradiol [26] are significantly higher during the secretion season than during the non-secretion season. We therefore speculate that the strong influence of testosterone and estradiol on musk secretion mainly occurs during the early stages of maturation. When we examined the relationship between fecal hormone levels and major musk components during the early phases of secretion, we found that testosterone and estradiol were significantly correlated with all four examined musk components, whereas at all other time points, only two or three components were 


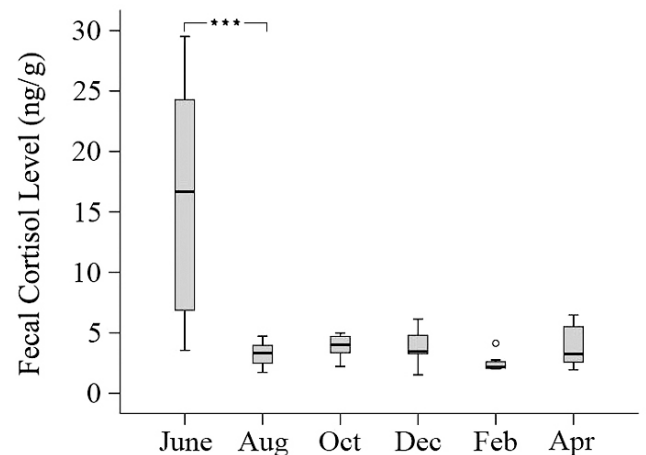

A. Testosterone

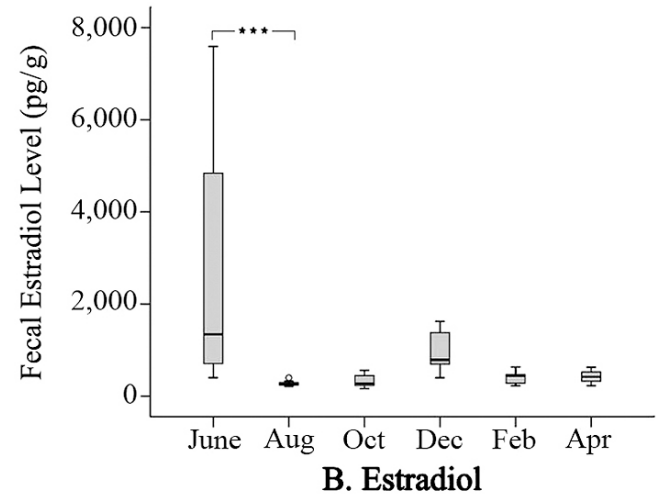

B. Estradiol

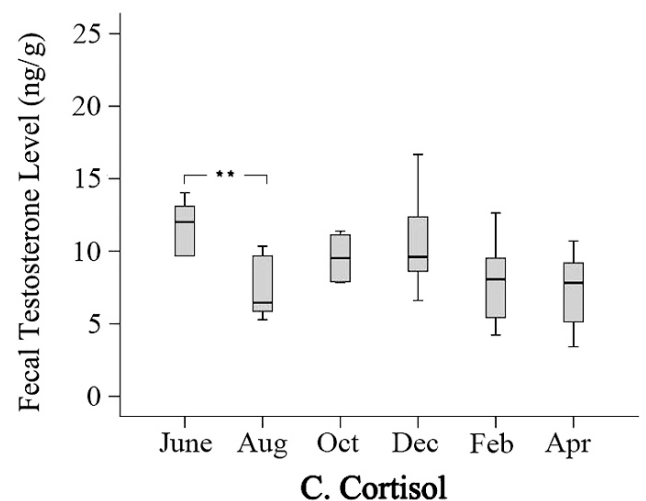

Fig. 5 Box plots of fecal hormone levels in Chinese forest musk deer across various months. X-axis: months (June 2016, August 2016, October 2016, December 2016, February 2017, April 2017); Y-axis: fecal hormone levels. The Y-axis units of A and C are ng $\mathrm{mL}^{-1}$ and the Y-axis unit of $\mathrm{B}$ is $\mathrm{pg} \mathrm{mL}^{-1}$. (A: Testosterone, B: Estradiol, $\mathrm{C}$ : Cortisol). ${ }^{\circ}$ indicates greater than 1.5 times and less than 3 times the interquartile range (IQR). $(*: p<0.05 ; * *: p<0.01 ; * * *: p<0.001)$.

significantly correlated with both these hormones (Tables 1 and 2). Testosterone and estradiol are secreted from distal sites, have prolonged activity times, and are feedback-inhibited [27]. Thus, although the levels of both hormones are high in June, their effects were not apparent. By August, however, the hormones were bioactive and there was a corresponding increase in the levels of active musk component. The effect of hormones on musk components may be delayed, which means that negative regulation then lowered hormone levels, resulting in a negative correlation with musk component levels. Analysis of the correlation between hormones and musk components indicates that the former play important roles during the early phases of active musk secretion, but do not significantly influence or participate in the latter stages of the musk maturation process.

There have been many studies on the secretion of volatile compounds and their dependency on hormone levels in other species. For example, the temporal gland secretions of Asian bull elephants have been shown to be
Table 1 Correlation analysis between levels of 4 major components in musk and testosterone levels in the feces of musk deer (A: June-August; B: August-April)

\begin{tabular}{|c|c|c|}
\hline \multicolumn{3}{|l|}{ A } \\
\hline Component & Correlation coefficient & Sig. (two-tailed) \\
\hline Muscone & $-0.743 * *$ & 0.000 \\
\hline Cyclopentadecanone & $-0.749 * *$ & 0.000 \\
\hline Cholesterol & $-0.750 * *$ & 0.000 \\
\hline Cholestanol & $-0.783 * *$ & 0.000 \\
\hline \multicolumn{3}{|l|}{ B } \\
\hline Component & Correlation coefficient & Sig. (two-tailed) \\
\hline Muscone & -0.015 & 0.910 \\
\hline Cyclopentadecanone & -0.146 & 0.280 \\
\hline Cholesterol & $0.295^{*}$ & 0.026 \\
\hline Cholestanol & $0.316^{*}$ & 0.017 \\
\hline
\end{tabular}

$* p<0.05, * * p<0.01$. 
Table 2 Correlation analysis between levels of 4 major components in musk and estradiol levels in the feces of musk deer (A: June-August; B: August-April)

\begin{tabular}{ccc}
\hline \multicolumn{1}{l}{ A } & & \\
\hline Component & Correlation coefficient & Sig. (two-tailed) \\
\hline Muscone & $-0.644^{* *}$ & 0.001 \\
Cyclopentadecanone & $-0.674^{* *}$ & 0.001 \\
Cholesterol & $-0.742^{* *}$ & 0.000 \\
Cholestanol & $-0.763^{* *}$ & 0.000 \\
\hline B & & \\
\hline \multirow{2}{*}{ Component } & Correlation coefficient & Sig. (two-tailed) \\
\hline Muscone & -0.061 & 0.651 \\
Cyclopentadecanone & -0.181 & 0.178 \\
Cholesterol & $0.278^{*}$ & 0.036 \\
Cholestanol & 0.143 & 0.288
\end{tabular}

$* p<0.05, * * p<0.01$.

positively correlated with fecal androgen levels but show no correlation with fecal cortisol levels during musth $[28,29]$. In female goats (Capra hircus), higher levels of estradiol during estrus and higher level of progesterone during the post-estrus period have been observed [30]. In blackbuck, fecal estrogen concentration is significantly higher during estrus than during the proestrus and diestrus periods, and fecal progesterone concentration is significantly higher during diestrus than during the proestrus and estrus periods [31]. These observations indicate that the secretion of volatile compounds is regulated by various sex hormones in different animal species. In addition to testosterone and estradiol, the effect of progesterone and other sex hormones on the secretions of musk deer and the hormonal regulatory mechanisms underlying these secretions warrant further in vivo and in vitro study in the future.

\section{Conclusions}

The form, water content, and composition of musk
Table 3 Correlation analysis between levels of 4 major components in musk and cortisol levels in the feces of musk deer (A: June-August; B: August-April)

\begin{tabular}{ccc}
\hline \multicolumn{1}{l}{ A } & & \\
\hline Component & Correlation coefficient & Sig. (two-tailed) \\
\hline Muscone & -0.337 & 0.171 \\
Cyclopentadecanone & -0.255 & 0.307 \\
Cholesterol & -0.287 & 0.249 \\
Cholestanol & -0.438 & 0.069 \\
\hline B & & \\
\hline \multirow{2}{*}{ Component } & Correlation coefficient & Sig. (two-tailed) \\
\hline Muscone & 0.220 & 0.122 \\
Cyclopentadecanone & 0.276 & 0.050 \\
Cholesterol & 0.256 & 0.070 \\
Cholestanol & 0.176 & 0.218
\end{tabular}

$* p<0.05, * * p<0.01$.

change over the course of the secretion period. Mature musk comprises characteristic components, and there are significant correlations between the changes in musk components and the levels of testosterone and estradiol during the incipient stage of secretion, whereas there are no significant correlations at other times, and cortisol levels appear to be uncorrelated with musk component contents during the entire secretory period. The sex hormones play promoting roles in musk composition during the early stages of musk secretion in musk deer.

\section{Acknowledgements}

This work was supported by Zhangzhou Pien Tze Huang Pharmaceutical Co. Ltd. (Grant No. 2015HXFWBHQLSQ-001) and the China Postdoctoral Science Foundation (Postdoctoral Grant No. 168033; Grant No. 2016 M591094). The experimental facilities were provided by the Innovation Laboratory of the College of Nature Conservation, Beijing Forestry University.

\section{References}

1. State Forestry Administration (2003) Wildlife under special state protection list. Beijing, State Forestry Administration (In Chinese).

2. Zhang BL, Deng FM, Li BS, Zhu DX, Chen SW (1979)
Domestication of Musk Deer. China Agriculture Press, Beijing: 77-78 (In Chinese).

3. Zheng SW, Pi NL (1984) Studies on musk gland, musk, numbers, and hunt of the musk deer. Acta Theriol Sin 4: 
35-42 (In Chinese).

4. Li FD, Hong YS, Deng WM, Li SW (1980) The secretory regulation of musk deer. J Chin Medic Mater 4: 12-18 (In Chinese).

5. Chen BL, Zhu Q, Li M, Li DY (2016) Studies on the chemical composition and microbial composition of musk deer. China Animal Husbandry Science and Technology (In Chinese).

6. Su GY, Wu AL, Gan XN, Yue BS, Li J (2009) Quantitative analysis of musk components by gas chromatography/ mass spectrometry. Sichuan J Zool 28: 509-512, 516 (In Chinese).

7. Zhang NR (1987) A study on musk and its substitutes. Chin Trad Herb Drug 18: 38-41 (In Chinese).

8. Li CT, Fu MN, Yu LY (1992) Musk, a medicine from animals. Chin J Wildl 6: 4-8 (In Chinese).

9. Dong WC, Zhao WG, Liu CH (2001) Recent advances in musk research. Spec Wildl Econ Anim Plant Res 2: 48-58 (In Chinese).

10. Yin SY, Dai WG (1990) The secondary musk secretion of male musk deer induced physiologically by exogenous androgen within successive three years. J Sichuan Univ Eng Sci Ed 5: 41-46 (In Chinese).

11. Yin SY, Dai WG (1991) A study on the secondary musk secretion of male musk deer induced physiologically by androgen within successive two years. Acta Theriol Sin 11: 9-12 (In Chinese).

12. Hong QS, Li FD, Deng WM, Li SW (1981) The relationship between musk secretion and androgen. Chin Med Mat 1: 19-22 (In Chinese).

13. Wang JL, Huang XM, Zhu DX (1982) Effect of gonadotropic hormone to the musk-secretion of musk-deer. Acta Theriol Sin 2: 105-106 (In Chinese).

14. Wang JL, Huang XH, Yang CJ, Zhu CS, Zhu DX (1986) Effect of LRH analog to musk secretion. Spec Wildl Econ Anim Plant Res 4: 1-3 (In Chinese).

15. Bi SZ, Yan YH, Jin SZ, Wu YM, Chen CF, et al. (1980) A preliminary study of hypothalamus- pituitary- testicular system regulates musk secretion and improve musk production. Chin Med Mat 4: 18-20 (In Chinese).

16. Monfort SL, Martinet C, Wildt DE (1991) Urinary steroid metabolite profiles in female Père David's deer (Elaphurus davidianus). J Zool Wildl Med 22: 78-85.

17. Morrow CJ, Monfort SL (1998) Ovarian activity in the scimitar-horned oryx (Oryx dammah) determined by faecal steroid analysis. Anim Reprod Sci 53: 191-207.

18. Terio KA, Brown JL, Moreland R, Munson L (2002) Comparison of different drying and storage methods on quantifiable concentrations of fecal steroids in the cheetah.
Zool Biol 21: 215-222.

19. Schwarzenberger F (2007) The many uses of non-invasive faecal steroid monitoring in zoo and wildlife species. Int Zoo Yearb 41: 52-74.

20. Yang Q, Meng X, Xia L, Feng Z (2003) Conservation status and causes of decline on musk deer (Moschus spp.) in China. Biol Conserv 109: 333-342.

21. Zhang JX, Sun L, Zhang JH, Feng ZY (2008) Sex and gonad-affecting scent compounds and 3 male pheromones in the rat. Chem Senses 33: 611-621 (In Chinese).

22. Zhang JX, Zhang ZL, Wang ZW (1999) Effects of testosterone on flank glands in ratlike hamsters, Cricetulus triton. Zool Res 20: 318-320 (In Chinese).

23. Novotny M, Jorgenson JW, Carwack M, Wilson SR, Boyse EA, et al. (1980) Chemical Studies of the Primer Mouse Pheromones. In: Müller-Schwarze D, Silverstein RM (eds) Chemical Signals. Springer, Boston, MA.

24. Hu BL, Yang YZ, Hu SQ, Zhu L (1997) Recent advances in musk research. Inf Trad Chin Med 2: 11-12 (In Chinese).

25. Bai K, Ren ZJ, Wang YQ, Li FR, Li Y, et al. (2013) An association with gonadal hormone level and musk yield in Moschus berezovskii during musk-secreting period. Chin J Vet Sci 33: 956-962 (In Chinese).

26. Zhang ZM, Yang J, Wang JM, Fu WL, Wang ZY, et al. (2015) Changes of serum gonadal hormones levels during musk-secreting period and estrus of Moschus berezovskii. Zhong Yao Cai 38: 240-244 (In Chinese).

27. Yang XP, Xiao XH (2009) Animal Physiology, second ed. Higher Education Press, Beijing: 283 (In Chinese) (Abstract).

28. Rasmussen LE, Hess DL, Haight JD (1990) Chemical analysis of temporal gland secretions collected from an Asian bull elephant during a four-month musth episode. $J$ Chem Ecol 16: 2167-2181.

29. Ghosal R, Ganswindt A, Seshagiri PB, Sukumar R (2013) Endocrine correlates of musth in free-ranging Asian elephants (Elephas maximus) determined by non-invasive fecal steroid hormone metabolite measurements. PLoS One 8: e84787.

30. SankarGanesh D, Ramachandran R, Muniasamy S, Saravanakumar VR, Suriyakalaa U, et al. (2014) A correlation of fecal volatiles and steroid hormone profiles with behavioral expression during estrous cycle of goat, Capra hircus. Gen Comp Endocrinol 206: 178-183.

31. Archunan G, Rajagopal T (2012) Detection of estrus in Indian blackbuck: behavioural, hormonal and urinary volatiles evaluation. Gen Comp Endocrinol 181: 156-166. 